\title{
Assessment of accuracy of genomic prediction for French Lacaune dairy sheep
}

\author{
G. Baloche, ${ }^{*}$ A. Legarra, ${ }^{\star}$ G. Sallé, †‡ H. Larroque, ${ }^{*}$ J.-M. Astruc, § C. Robert-Granié, ${ }^{\star}$ and F. Barillet ${ }^{* 1}$ \\ *INRA, UR631, Station d'Amélioration Génétique des Animaux (SAGA), CS-52627, F-31326 Castanet-Tolosan, France \\ †INRA, UMR1282 Infectiologie et Santé Publique, F-37380 Nouzilly, France \\ łUniversité François Rabelais de Tours, UMR1282 Infectiologie et Santé Publique, F-37000 Tours, France \\ §Institut de l'Elevage, 149 rue de Bercy, F-75595 Paris, France
}

\begin{abstract}
Genomic selection in Lacaune dairy sheep was investigated based on genotypes from the OvineSNP50 BeadChip (Illumina Inc., San Diego, CA). Historical artificial insemination progeny-tested rams formed a population of 2,892 genotyped rams. Additional ungenotyped rams and females were included by singlestep genomic BLUP (ssGBLUP). Three prediction strategies were tried: pseudo-BLUP (using all rams and daughter yield deviations), pseudo-ssGBLUP (using all rams and daughter yield deviations), and regular ssGBLUP (using all phenotypes and pedigree in an animal model). The population linkage disequilibrium was determined, with an average squared correlation coefficient of 0.11 for markers closer than $0.1 \mathrm{cM}$ (lower than in dairy cattle). The estimated effective population is 370 individuals. Gain in accuracy of genomic selection over parent averages ranged from 0.10 to 0.20 . Highest accuracies and lowest bias were found using regular ssGBLUP. Transition to a genomic breeding scheme is possible but costs need to be carefully evaluated.
\end{abstract}

Key words: genomic selection, Lacaune dairy sheep, accuracy

\section{INTRODUCTION}

Accurate genomic predictions that combine genotypic, phenotypic, and pedigree data available early in the life of livestock have the potential to reduce the generation interval for breeding schemes that focus on progeny testing (Meuwissen et al., 2001; Schaeffer, 2006). However, the potential reduction of generation interval for Lacaune dairy sheep is limited because the use of AI with fresh semen and a very rapid turnover of males result in an already short generation interval of 4.2 yr. However, early selection of fewer rams might

Received June 13, 2013.

Accepted October 22, 2013.

${ }^{1}$ Corresponding author: francis.barillet@toulouse.inra.fr decrease the maintenance costs of AI rams that are currently waiting for first-crop progeny tests.

The Lacaune dairy sheep breed was defined in the 1950 s to 1960s by the pooling of several local breeds (Quittet and Franck, 1983). Genetic improvement programs for Lacaune dairy sheep started in the 1960s and have been managed by 2 AI companies [Conféderation Générale de Roquefort (Millau, France) and Ovitest (Onet-le-Château, France)] since 1972. They progeny test 470 rams annually in a nucleus group of 368 commercial flocks (i.e., the companies are AI centers that do not own the flocks), with around 170,000 ewes recorded yearly for several traits. Those nucleus flocks, which are at the top of a pyramidal organization and include $20 \%$ of the Lacaune population (Barillet et al., 1986), benefit from extensive performance recording and AI. This is important because, contrary to dairy cattle, performance recording for sheep has high associated costs.

In practice, the existence of 2 companies implies 2 little-related subpopulations (which can be clearly seen using, for example, principal components analysis). Modeling the 2 subpopulations as a single one or as separate ones did not significantly change the results of this work (results not shown). That is, similar accuracies were observed when validation was performed within each subpopulation or the joint data set (results not shown).

Extensive AI for sheep is accomplished through the use of fresh semen, which requires the availability of numerous living rams to face seasonal AI demands that peak, in Lacaune, at 26,000 inseminations per week per company. Therefore, there is no storage of frozen semen. Thus, collection and storage of blood samples of AI rams began in 1995 in the view of QTL detection and localization and marker-assisted selection.

To develop a dense SNP chip for sheep, 20 countries organized the International Sheep Genomics Consortium (http://www.sheephapmap.org) in 2002. The OvineSNP50 BeadChip (Illumina Inc., San Diego, CA) was finally released in 2009. 
Recently, a large set of Lacaune rams has been genotyped, with a focus on QTL detection and genomic selection. Duchemin et al. (2012) compared methods for genomic selection of Lacaune dairy sheep and reported an absolute increase in accuracy of 0.05 to 0.10 as assessed by forward validation compared with parent average. They used the same data for the reduced and full data sets as defined by Interbull (Mäntysaari et al., 2010), which overestimates accuracy (Olson et al., 2011) and makes the benefit of genomic selection for candidates unclear.

In addition, linkage disequilibrium (LD; the nonrandom association of alleles between 2 loci) was investigated because it strongly influences the power of QTL detection and the accuracy of genomic prediction (Pritchard and Przeworski, 2001; Ober et al., 2012). According to Meuwissen et al. (2001), genomic predictions reach an accuracy of 0.85 with a simulated LD of 0.20 . Such a level of LD was hypothesized to be achieved with 35,000 multiallelic markers (Meadows et al., 2008) for meat and wool. However, Kijas et al. (2012) reported lower LD with the commercial OvineSNP50 BeadChip, which achieves such a density.

A factor related to $\mathrm{LD}$ is the effective population size: the smaller the population size, the higher the disequilibrium and the expected accuracy of linkage disequilibrium (Goddard, 2009). The latest estimates of effective population size in Lacaune sheep are pedigree based (Palhiere et al., 2000) and outdated.

The objectives of this study were to provide consolidated and updated LD measures across SNP markers and to determine the accuracy of genomic predictions through forward validation in the Lacaune breed. Concerning estimation of accuracy, this study differs from that of Duchemin et al. (2012) in the use of singlestep genomic BLUP (ssGBLUP; Aguilar et al., 2010; Christensen and Lund, 2010), in the use of reduced and full data sets to assess accuracy by forward validation, and in a larger data set.

\section{MATERIALS AND METHODS}

\section{Genomic Data}

Currently, 5,000 progeny-tested rams with more than 10 daughters have DNA stored. Because exhaustive genotyping of rams was too expensive, the following genotyping strategy was chosen. Rams born from 2008 through 2009 and progeny tested were constituted as the validation population (see Table 1). Then, complete generations were (as much as possible) genotyped backward, including most rams born from 1998 through 2007 to form the training population. These included most ancestors (sires and maternal grandsires) of the
Table 1. Distribution of Lacaune dairy rams in the genomic selection test

\begin{tabular}{lcc}
\hline Population & Rams (no.) & Birth year \\
\hline Training & 1,593 & $1999-2005$ \\
Excluded & 707 & $2006-2007$ \\
Validation & 592 & $2008-2009$ \\
\hline
\end{tabular}

validation population. No ram with less than 20 daughters in progeny testing was genotyped.

Extraction of DNA from blood samples and genotyping was conducted by the LABOGENA laboratory (Jouy-en-Josas, France). Extracted DNA was available for $90 \%$ of the AI rams born from 2003 to 2009 and $25 \%$ for rams born from 1998 through 2002. Genotyped rams were roughly structured in 452 half-sib families, with a mean of 9 sons. Both breeding companies contributed to samples in accordance with the number of rams enrolled in their progeny-testing programs. The Illumina OvineSNP50 BeadChip developed through the International Sheep HapMap project (International Sheep Genomics Consortium, 2010) was used for genotyping. Illumina GenomeStudio software was used with default thresholds and cluster definitions for genotype calling.

From the set of SNP markers available, $92 \%$ were read for $99 \%$ of rams. Data for some SNP were discarded because of low minor allele frequency $(<0.01)$, HardyWeinberg disequilibrium $\left(P<10^{-5}\right)$, or insufficient genotyping rate $(<0.97)$. Mendelian inconsistencies were set to missing. Missing genotypes and genotyping errors $(0.25 \%)$ were imputed using BEAGLE v3.4 software (Browning and Browning, 2007). The final data set included genotypes of 42,039 SNP from 2,892 rams born from 1996 through 2009 (Figure 1).

\section{Phenotypes}

For training and validation populations, phenotypes were daughter yield deviations (DYD; VanRaden and Wiggans, 1991), which were weighted by equivalent daughter contribution (EDC; Fikse and Banos, 2001; Sullivan, 2007). The DYD and EDC were used as the variable response to compute genomic predictions from a pseudo-ssGBLUP or pedigree-based prediction from a pseudo-BLUP that will be detailed later. Alternatively, observed performance measures (as used in official evaluation), were used in ssGBLUP. Training and validation populations were defined according to Interbull (Uppsala, Sweden) rules (Mäntysaari et al., 2010) for both methods. This defines a reduced data set and a full data set to fairly predict genetic merit of validation rams. 


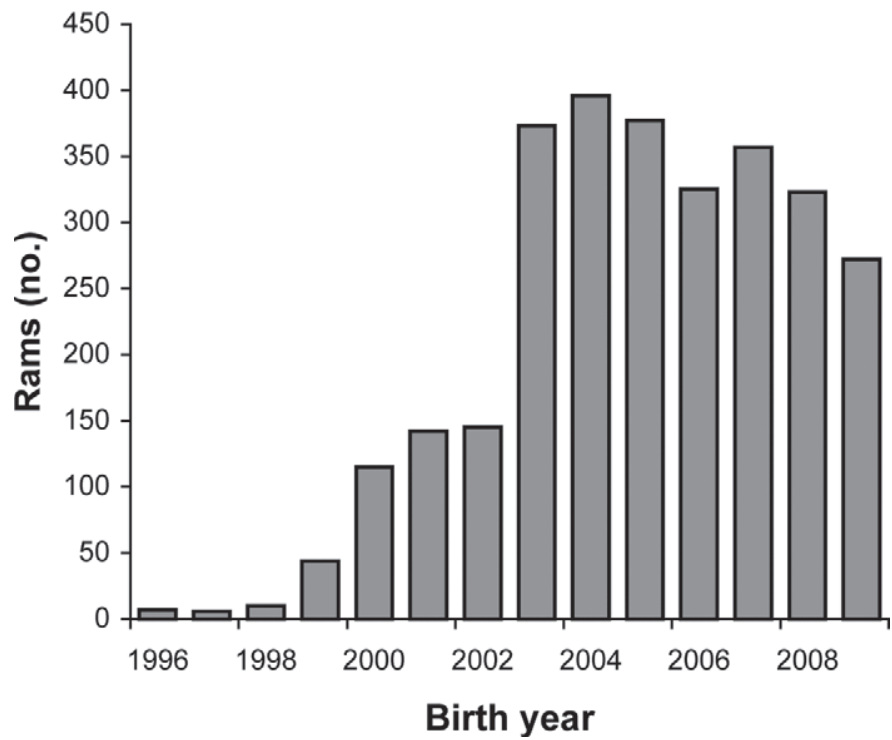

Figure 1. Distribution of AI genotyped rams $(\mathrm{n}=2892)$ by birth year.

The full data set (all data available in 2011) was required to compute reliable EBV estimates for the validation population. More than 4,431,000 phenotypic records were available for 1,436,000 animals. For the reduced data set, data from 2008 through 2011 was excluded from the full data set (i.e., the reduced data set included records available for genetic evaluation in 2007). The reduced data set contained 3,822,000 phenotypic records for 1,262,000 animals. Prediction was based on rams born up to 2005, whereas validation was based on rams born after 2007 (Table 1). Rams born in 2006 or 2007 ( $24 \%$ of rams) were excluded from the test to comply with Interbull rules $(<25 \%$; Mäntysaari and Koivula, 2012).

In addition to genotyped rams, 5,904 nongenotyped $\mathrm{AI}$ and natural-mating rams with progeny and born from 1990 through 2005 were included in the prediction step via pseudo-BLUP or ssGBLUP. Figure 2 shows the proportion of genotyped to nongenotyped rams by birth year from 1996 through 2009. Between 1990 and 1996, only nongenotyped rams were included.

Traits evaluated included milk yield, fat and protein percentages, SCS, and 3 udder traits (teat angle, udder cleft, and udder depth; Barillet, 1997; Marie-Etancelin et al., 2005; Barillet et al., 2007). Respective heritabilities were $0.30,0.45,0.35,0.13,0.33,0.26$, and 0.19 .

\section{Estimation of LD, Effective Population Size, and Expected Accuracy of Genomic Predictions}

The degree of LD was estimated using the squared correlation coefficient $\left(\boldsymbol{r}^{2}\right)$ statistic of Hill and Robert- son (1968). Computations were carried out between all SNP pairs in 10-Mbp windows within chromosome, as a correlation measure (Rogers and Huff, 2009). The $r^{2}$ were grouped into categories based on distance between SNP from the physical map.

The subsequent step consisted of comparing molecular data $\left(r^{2}\right)$ results with a pedigree-based estimator of the population diversity. Sved (1971) formulated that the approximate expectation of $r^{2}$ is $1 /\left(4 N_{e} c+1\right)$, where $N_{e}$ is the effective population size and $c$ is the recombination distance in morgans between SNP. Further, LD at short distance is dependent on long-term population history (Hill, 1981; Hayes et al., 2003). Consequently, the expectation of $r^{2}$ is $1 /\left(4 N_{e} c+1\right)$, where $N_{e}$ was the population size $1 /(2 c)$ generations ago (Hayes et al., 2003). The $N_{e}$ for $c=10 \mathrm{Mbp}$ was considered to be the most recent $N_{e}$ (5 generations ago).

The pedigree-based estimators of $N_{e}$ were the realized effective size, defined as $N_{e}=\frac{1}{2 \overline{\Delta F}}$, where $\overline{\Delta F}$ is the average of individual increase in inbreeding. Individual increase in inbreeding was computed following the definition of Gutiérrez et al. (2008): $\Delta F_{i}=1-\sqrt[t_{i}]{1-F_{i}}$. Individual inbreeding $\left(F_{i}\right)$ and $t_{i}$, the equivalent of discrete generation (Boichard et al., 1997), were computed using PEDIG software (Boichard, 2002). A subpedigree of 1,636,751 animals born from 1999 through 2010 and all their ancestors for 11 generations resulted in a total of $1,947,402$ animals.

Availability of $N_{e}$, heritabilities of traits, and size of the training population make possible to predict the

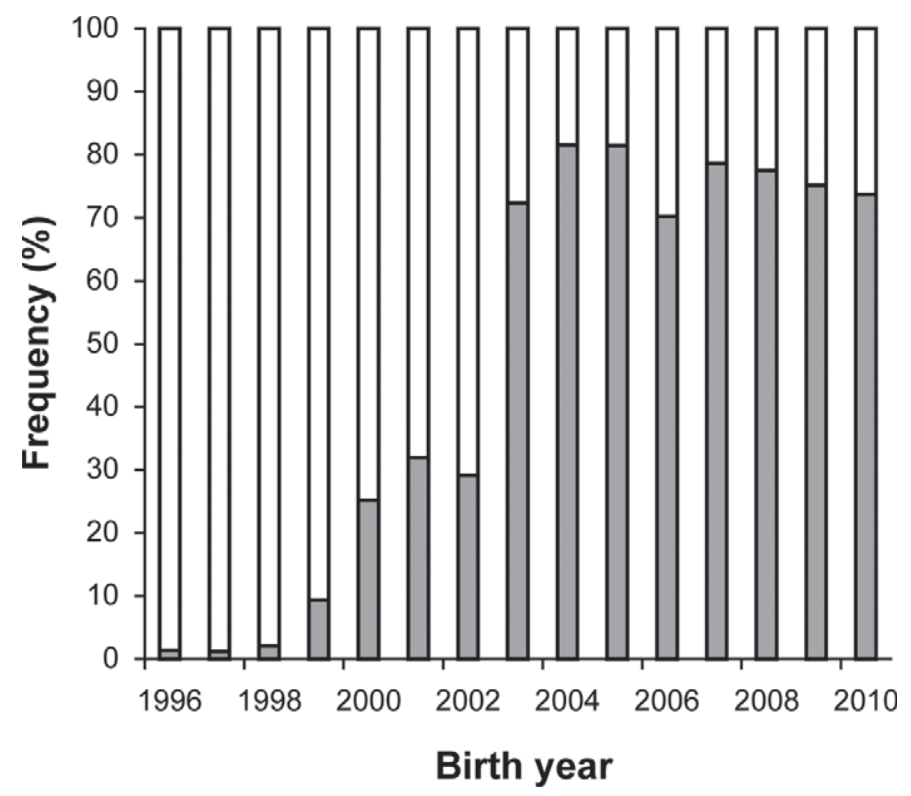

Figure 2. Proportion of genotyped rams (gray) among all AI rams included in the genomic test by birth year. 
expected accuracy of future genomic predictions. According to Daetwyler et al. $(2010), r_{g \hat{g}}=\sqrt{\frac{N_{P} h^{2}}{N_{P} h^{2}+M_{e}}}$, where $r_{g \hat{g}}$ is the correlation between true and estimated genetic values, $N_{P}$ is the number of ewes and rams phenotyped, $h^{2}$ is the heritability of the trait, and $M_{e}$ is the effective number of loci (Goddard, 2009).

\section{Genomic Evaluation}

Prediction Methods. Because of the results of Duchemin et al. (2012), who did not observe any improvement using either nonparametric or Bayesian methods such as BayesCPi, only BLUP-like methods were considered. Production traits were evaluated with a single-trait animal model with permanent environmental effects. Heterogeneous variances among herds were also considered for production traits (similar to the French dairy cattle model; Robert-Granié et al., 1999), except for ssGBLUP. Udder traits were evaluated using a multitrait animal model (Marie-Etancelin et al., 2005).

For pseudo-BLUP, phenotypes were DYD, and the pedigree-based relationship matrix included genotyped and nongenotyped rams. The "pseudo" name indicates that we did not use either a true BLUP animal model or parent average. Therefore, the model for pseudoBLUP was $\mathbf{y}_{\text {DYD }}=\mathbf{1} \mu+\mathbf{W} \mathbf{u}_{\text {rams }}+\varepsilon$, where $\mathbf{y}_{\text {DYD }}$ are DYD for each ram; $\mu$ is the overall mean; $\mathbf{W}$ is the incidence matrix allocating DYD to the breeding values; $\mathbf{u}_{\text {rams }}$ are breeding values, and it was assumed that $\operatorname{Var}\left(\mathbf{u}_{\text {rams }}\right)=\mathbf{A}_{\text {rams }} \sigma_{u}^{2}$, where $\mathbf{A}_{\text {rams }}$ is the numerator relationship matrix across rams and $\sigma_{u}^{2}$ is the genetic variance; and $\varepsilon$ is a vector of residuals. Pseudo-residual variances of $\varepsilon$ were weighted by the inverse of the EDC number for each DYD.
For pseudo-ssGBLUP, the model was as above, but it was assumed that $\operatorname{Var}\left(\mathbf{u}_{\text {rams }}\right)=\mathbf{H}_{\text {rams }} \sigma_{u}^{2}$, where $\mathbf{H}_{\text {rams }}$ is the combined pedigree and genomic relationship matrix described by Legarra et al. (2009), across all rams, as done by Gao et al. (2012). Finally, ssGBLUP included all animals using the following animal model: $\mathbf{y}=\mathbf{X b}$ $+\mathbf{W}_{u} \mathbf{u}\left(+\mathbf{W}_{p} \mathbf{p}\right)+\mathbf{e}$, which includes observed performances (i.e., ewe's records; $\mathbf{y}$ ), a matrix $\mathbf{X}$ relating the fixed effects (b) to the performance, whole-population breeding values $(\mathbf{u})$, permanent effects $(\mathbf{p})$ for the respective traits, and the residuals e. This model included all genotyped and nongenotyped animals (rams and sheep) and a joint genomic-pedigree relationship matrix (Legarra et al., 2009; Aguilar et al., 2010; Christensen and Lund, 2010) $\operatorname{Var}(\mathbf{u})=\mathbf{H} \sigma_{u}^{2}$. The variance among herds in regular genetic evaluation was ignored in ssGBLUP. Each scenario is detailed in Table 2.

For pseudo-BLUP and pseudo-ssGBLUP, only direct results were considered without any blending with parent average (VanRaden et al., 2009). This blending is automatically done in ssGBLUP. Both ssGBLUP methods used the genomic relationship matrix $(\mathbf{G})$ constructed as $\mathbf{G}=0.95 \mathbf{Z Z}^{\prime} / \sum 2 p_{i} q_{i}+0.05 \mathbf{A}_{22}$, where $\mathbf{Z}$ is the incidence matrix of marker effects, corrected by the observed genotype frequencies, $p_{i}$ is allele frequencies, $q_{i}=1-p_{i}$, and $\mathbf{A}_{22}$ is the matrix of pedigree relationship of genotyped animals obtained from the whole pedigree (VanRaden, 2008). These weights make $\mathbf{G}$ invertible and imply that $95 \%$ of the genetic variance is explained by markers. The matrix $\mathbf{G}$ was then corrected using BLUP $F_{F_{\mathrm{ST}}}$ (where $F_{\mathrm{ST}}$ is defined as the mean relationship between gametes in a recent population with respect to an older base population) as in Vitezica et al. (2011) to make it compatible with pedigree relationships. The programs PREGSf90 (computation of needed matrices), BLUPF90 (storage of mixed-model equa-

Table 2. Description of each scenario tested

\begin{tabular}{|c|c|c|c|}
\hline $\begin{array}{l}\text { Prediction } \\
\text { strategy }^{1}\end{array}$ & Phenotype $^{2}$ & $\begin{array}{l}\text { Relationship } \\
\text { matrix }^{3}\end{array}$ & Model $^{4}$ \\
\hline $\begin{array}{l}\text { Pseudo-BLUP } \\
\text { Pseudo-ssGBLUP } \\
\text { ssGBLUP }\end{array}$ & $\begin{array}{l}\text { 7,497 rams with } \mathrm{DYD}^{5} \text { (ignoring genotypes) } \\
7,497 \text { rams with DYD } \text { (including } 1,593 \text { genotyped training rams) }_{3,822,000 \text { performances; } 1,262,000 \text { animals in pedigree (including }} \\
1,593 \text { genotyped training rams) }\end{array}$ & $\begin{array}{l}\mathbf{A}_{\text {rams }} \\
\mathbf{H}_{\text {rams }} \\
\mathbf{H}_{\text {ewes+rams }}\end{array}$ & $\begin{array}{l}\mathbf{y}_{\text {DYD }}=\mathbf{1} \mu+\mathbf{W}_{1} \mathbf{u}_{\mathrm{rams}}+\varepsilon_{1} \\
\mathbf{y}_{\mathrm{DYD}}=\mathbf{1} \mu+\mathbf{W}_{2} \mathbf{u}_{\mathrm{rams}}+\varepsilon_{2} \\
\mathbf{y}=\mathbf{X b}+\mathbf{W}_{u} \mathbf{u}+\mathbf{W}_{p} \mathbf{p}+\mathbf{e}\end{array}$ \\
\hline
\end{tabular}

${ }^{1}$ ssGBLUP $=$ single-step genomic BLUP.

${ }^{2} \mathrm{DYD}=$ daughter yield deviations.

${ }^{3} \mathbf{A}_{\text {rams }}=$ numerator relationship matrix across rams; $\mathbf{H}_{\text {rams }}=$ combined pedigree and genomic relationship matrix described by Legarra et al. (2009), across all rams; $\mathbf{H}_{\text {ewes+rams }}=$ combined pedigree and genomic relationship matrix described by Legarra et al. (2009), across all ewes and rams.

${ }^{4} \mathbf{y}_{\mathrm{DYD}}=$ DYD for each ram; $\mu=$ overall mean; $\mathbf{W}=$ incidence matrix allocating DYD to the breeding values; $\mathbf{u}_{\mathrm{rams}}=$ breeding values; $\boldsymbol{\varepsilon}=$ vector of residuals; $\mathbf{y}=$ ewe's records; $\mathbf{X}=$ incidence matrix relating the fixed effects to the performances; $\mathbf{b}=$ fixed effects; $\mathbf{u}=$ whole-population breeding values; $\mathbf{p}=$ permanent effects; $\mathbf{e}=$ the residuals.

${ }^{5}$ Progeny tested.

${ }^{6}$ Including the 7,497 rams above (1,593 of them genotyped). 
tions), and BLUP90IOD2 (iteration on data) from the BLUPF90 program family were used (Misztal et al., 2002; Aguilar et al., 2010, 2011).

The derivation of expected reliability was by sparse inversion of the mixed-model equations in pseudo-BLUP and pseudo-ssGBLUP. However, expected reliabilities could not be computed for the reduced data set used in ssGBLUP, which had 1,254,767 animals in pedigrees and $3,738,475$ phenotypic records. Those reliabilities were obtained using the approximation described by Misztal et al. (2013). Computing time is negligible $(<2 \mathrm{~h}$ for the complete evaluation in all cases) and is mostly the result of the creation of dense matrices for ssGBLUP.

Prediction Accuracy. The accuracy of genomic predictions was tested using the methods of Mäntysaari et al. (2010). Regardless of method (ssGBLUP or pseudo-ssGBLUP), pseudo-phenotypes (DYD) of the validation rams obtained from the full data set were taken as a surrogate variable for true breeding value. Different estimators of breeding values were compared with DYD using regression: DYD $=b_{0}+b_{1} \mathrm{EBV}+e$, where $b_{0}$ is the intercept, $b_{1}$ is the slope, and $e$ is the residual. The coefficient of determination $\left(R^{2}\right)$ was the reliability of the prediction. The realized reliability (accuracy) was adjusted ( $a d j)$ to take into account the reliability of the surrogate variable (Mäntysaari et al., 2010): $\mathrm{R}_{a d j}^{2}=\mathrm{R}^{2}(1+k / \overline{E D C})$, where $k=\left(4-h^{2}\right) / h^{2}$ and $\overline{E D C}$ is the average EDC over the validation population. Selective genotyping (Mäntysaari and Koivula, 2012) has an influence on the expected slope but did not occur in our data set.

Effect of Mean Relationship on Genomic Accuracy. As demonstrated by Habier et al. (2010), the degree of relationship between the training set and selection candidates affects prediction accuracy. As indicated in a preliminary investigation of a genomic breeding scheme for Lacaune sheep (Buisson et al., 2013), in a fast genomic scheme, a fraction of candidates will have sires that will have no progeny test or daughter performance. Therefore, the effect of removing from the training data set the sires of individuals in the validation data set was tested using pseudo-ssGBLUP as described previously.

\section{RESULTS}

\section{$L D$}

For the 42,039 SNP for 2,892 rams included in the genomic selection test, the mean minor allele frequency was $0.29 \pm 0.13$. All possible SNP pairs with a distance of $<10 \mathrm{Mbp}$ within chromosome produced 6,859,395 pairwise $r^{2}$ on the 26 ovine autosomes with pairs. To

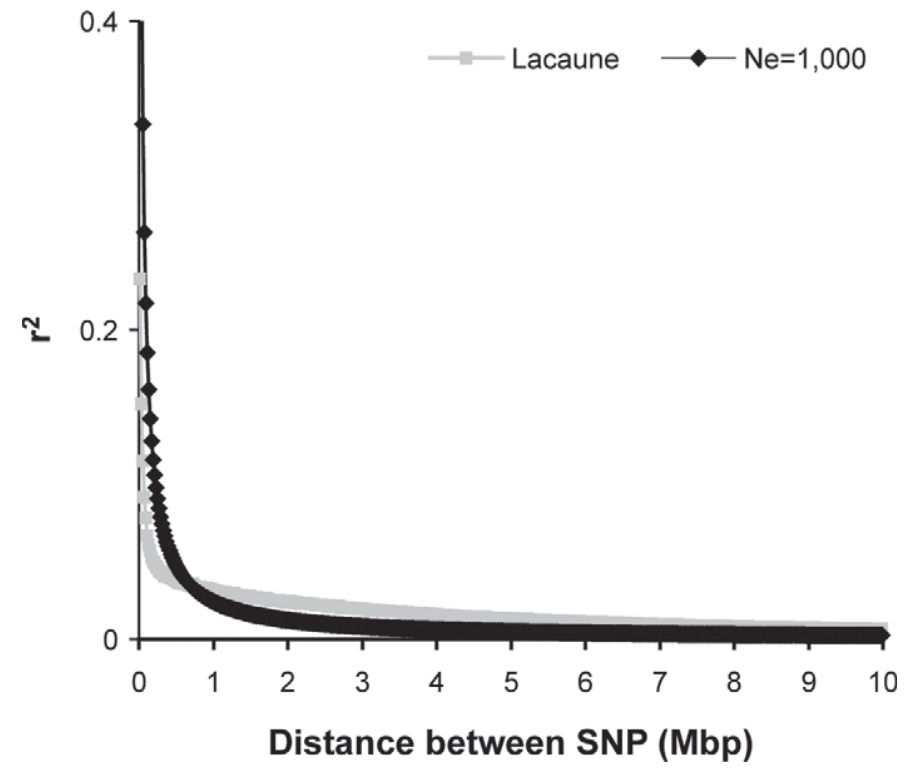

Figure 3. Decay in linkage disequilibrium measured as squared correlation coefficient $\left(r^{2}\right)$ by distance between SNP. $N_{e}=$ effective population size.

visualize the decay of $\mathrm{LD}, r^{2}$ were averaged within category of intermarker spacing $(0.02 \mathrm{Mbp})$ and plotted against that intermarker distance (Figure 3). The observed pattern shows LD in inverse relationship with genetic distance as regularly observed in cattle (e.g., de Roos et al., 2008).

Given that the map coverage of the informative SNP reached 2,419 $\mathrm{cM}$, the mean SNP interval was 0.056 $\mathrm{cM}$. Assuming that $1 \mathrm{cM}=1 \mathrm{Mbp}$, the mean $r^{2}$ was $0.11 \pm 0.16$ for a distance of $<0.1 \mathrm{cM}$ and $0.13 \pm 0.18$ for a marker spacing of $<0.05 \mathrm{cM}$. A similar study by Kijas et al. (2012) reported similar LD for dairy and meat Lacaune sheep (103 individuals). The SNP-based LD estimates were lower than those of Meadows et al. (2008), who reported that LD for an intermarker distance of $1 \mathrm{cM}$ could reach 0.1 .

Compared with LD decay for a population with $N_{e}$ $=1,000, r^{2}$ for Lacaune sheep was lower for small SNP intervals and higher for large intervals (Figure 3). According to Sved (1971), the LD for small SNP intervals indicates that the Lacaune sheep had broad diversity $\left(N_{e}\right.$ of $\left.>1,000\right) 5,000$ generations ago. Intense selection later led to high LD for large SNP intervals.

\section{Effective Population Size}

Plotting the effective population at various generations (Figure 4) showed a linear decrease of $N_{e}$ from 6,000 individuals 5,000 generations ago to 3,000 individuals 500 generations ago. Then, $N_{e}$ was reduced 10fold with a 2 -fold reduction, from 800 to 370 individuals 
1112

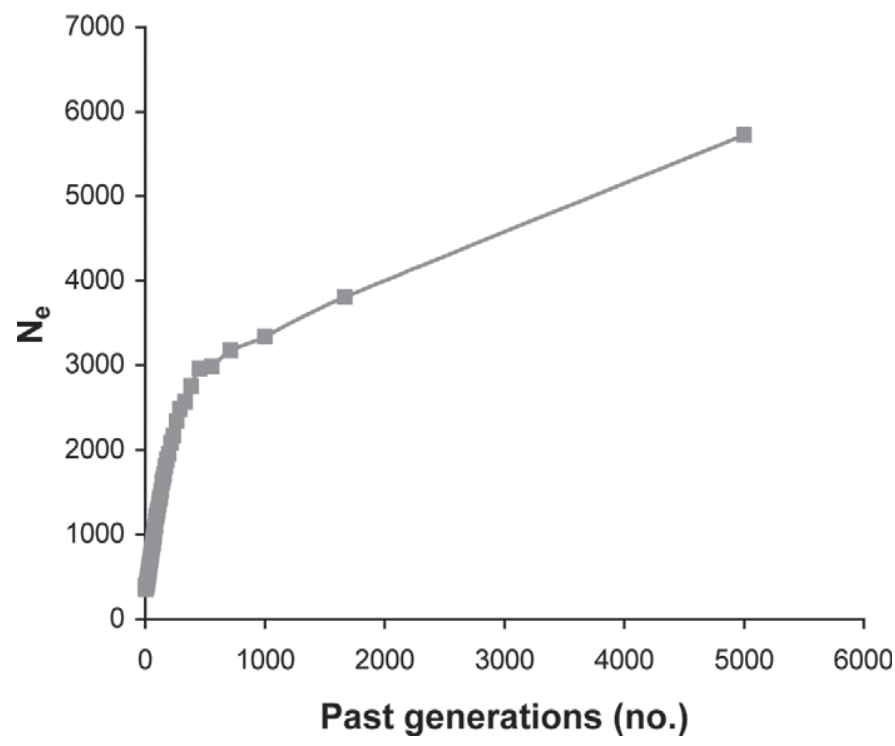

Figure 4. Effective population size $\left(\boldsymbol{N}_{e}\right)$ for the last 5,000 generations of Lacaune dairy sheep.

in the last 50 generations. This confirms the reduction on effective size due to the definition of the Lacaune standards after the pooling of small local breeds and last, the intense dairy selection. Based on pedigree, the mean number of known ancestors was 5.41 (ranged from 0.5 to 9.42 ) and the realized effective population size is 192 . Considering an older population (5 generations ago) to be fully comparable with the LD estimate, $N_{e}$ reached 252. Those figures are remarkably close to the estimate based on markers.

\section{Genomic Predictions}

Using the formula of Daetwyler et al. (2010), the expected accuracy of genomic prediction across traits is, on average, 0.57. Parameters used were a $N_{e}$ of 370 , a training population composed of 1,593 rams genotyped. Progeny testing was supposed to include 40 daughters per ram.

The observed accuracy for different scenarios of genomic evaluation is listed in Table 3 . The accuracy of genomic predictions from pseudo-ssGBLUP and ssGBLUP was around 0.6 to 0.7 , regardless of trait heritability, except for accuracy around 0.45 for milk yield. The pseudo-BLUP accuracy was 0.12 lower, on average, for all traits. Accuracy gain (genomic minus pedigree) was maximal for udder cleft $\left(h^{2}=0.23\right)$ and minimal for fat percentage $\left(h^{2}=0.35\right)$. Molecular data roughly enhanced parent-average accuracy by 0.10 . In regard to regression slopes (Table 4), pseudo-ssGBLUP outperformed pseudo-BLUP (i.e., slopes were closer to 1.00) except for SCS. Single-step GBLUP clearly improved slopes, except for fat percentage and SCS.

The exclusion of the 93 sires of validation rams from the training data set decreased the size of the training set by $6 \%$. Based on the entire genomic relationship matrix, reduction of the mean genomic relationship between the training and validation sets was limited to $0.48 \%$. Also, the maximum genomic relationship coefficient was 0.46 instead of 0.65 . Forward validation accuracy decreased in the validation set for all traits (Table 5). The accuracy loss was severe for udder depth, teat angle, and SCS (0.12-0.13) and moderate for milk yield (0.05). Applying the same truncation to pseudoBLUP led to an even higher (0.20) loss of accuracy. Hence, this confirms that a selection scheme with rapid turnover of males is more efficient using genomic than pedigree information.

\section{DISCUSSION}

\section{Population Structure}

A similar pattern of $\mathrm{LD}$ along genomic distances was described by de Roos et al. (2008) although LD was clearly lower in our case. Within the ovine species, the $r^{2}$ of 0.11 at $<0.1 \mathrm{cM}$ found in the current study confirmed observations of Kijas et al. (2012) based on 103 Lacaune dairy sheep. However, as $r^{2}$ tends to be biased upward for low minor allele frequencies, the threshold of 0.01 minor allele frequency in the current study (versus 0.10 in Kijas et al., 2012) might have led to underestimated $r^{2}$. Such low LD levels suggest that the Lacaune sheep come from highly heterogeneous

Table 3. Accuracies obtained with pseudo-BLUP, pseudo-single-step genomic BLUP (pseudo-ssGBLUP), or ssGBLUP

\begin{tabular}{|c|c|c|c|c|c|}
\hline Trait & \multicolumn{3}{|c|}{ Prediction method } & \multicolumn{2}{|c|}{ Accuracy gain over pseudo-BLUP } \\
\hline Milk yield & 0.32 & 0.43 & 0.47 & 0.11 & 0.15 \\
\hline Protein percentage & 0.54 & 0.62 & 0.70 & 0.08 & 0.16 \\
\hline SCS & 0.49 & 0.59 & 0.59 & 0.10 & 0.10 \\
\hline Teat angle & 0.47 & 0.58 & 0.66 & 0.11 & 0.19 \\
\hline
\end{tabular}


Table 4. Slopes for regression of predictions from pseudo-BLUP, pseudo-single-step genomic BLUP (pseudossGBLUP), or ssGBLUP on daughter yield deviations

\begin{tabular}{lccc}
\hline & \multicolumn{3}{c}{ Method } \\
\cline { 2 - 4 } Trait & Pseudo-BLUP & Pseudo-ssGBLUP & ssGBLUP \\
\hline Milk yield & 0.63 & 0.70 & 0.75 \\
Fat percentage & 1.10 & 1.00 & 0.93 \\
Protein percentage & 0.85 & 0.86 & 0.90 \\
SCS & 0.81 & 0.74 & 0.73 \\
Teat angle & 0.83 & 0.87 & 0.95 \\
Udder cleft & 0.72 & 0.77 & 0.81 \\
Udder depth & 0.92 & 0.94 & 0.97 \\
\hline
\end{tabular}

populations because 500 generations ago $N_{e}$ was 3,000 , whereas the current $N_{e}$ confirmed by a pedigree-based approach was between 200 (contemporary population) and 370 individuals ( 5 generations ago). Most of the breeds that Kijas et al. (2012) studied had a similar LD pattern and level. Compared with dairy cattle, the steeper LD trajectory in Lacaune sheep sheds light on moderate selection and bottlenecks. Although LD was not as favorable as in dairy cattle (de Roos et al., 2008; Sargolzaei et al., 2008), it should be sufficient for estimating accurate genomic breeding values (Goddard, 2009).

\section{Genomic Versus Pedigree Prediction Accuracy}

Accuracy gain of genomic predictions over parent average $(0.12$, on average) was limited compared with that for common dairy cattle breeds $(0.05-0.25$; VanRaden et al., 2009) and with predictions by Daetwyler et al. (2010). The smaller gain might result from the following reasons: lower level of LD, lower DYD accuracy (or, equivalently, less information per genotyped animal), lower coverage of old history (essentially all bulls are genotyped in Holsteins, whereas this is not the case in the current work), and less reliable forward validation because of the less accurate EBV in the full data set. In addition, higher reliability for pseudo-
BLUP predictions than in dairy cattle was caused by beneficial aspects of data structure, such as the large size of contemporary groups in large sheep flocks and lack of preferential treatments (Duchemin et al., 2012).

Beyond accuracies, slopes of DYD versus genomic predictions were of major importance. A slope $>1$ indicated underdispersion of genomic predictions, whereas a slope $<1$ indicated overdispersion. In this study, although pseudo-ssGBLUP was computed taking into account DYD of nongenotyped rams, ssGBLUP gave better results for slopes, which agrees with the findings of Aguilar et al. (2010) and Gao et al. (2012). Enhanced fit with ssGBLUP results from including more and less biased information. In particular, ssGBLUP considers information from dams of candidates (or, in other words, full parent average), which is not the case in pseudo-ssGBLUP, where the maternal information is plainly ignored (although the maternal grandsire is considered). In dairy cattle, either using selection index to combine sources of information (genomic proofs + parent averages; VanRaden et al., 2009), or using a single step gives similar results (Aguilar et al., 2010), and in fact the single step can be seen as an iterated selection index (Aguilar et al., 2010; Legarra and Ducrocq, 2012).

Presently, using a matrix that includes both pedigreebased relationships and differences between pedigree

Table 5. Accuracies obtained with pseudo-BLUP and pseudo-single-step genomic BLUP (pseudo-ssGBLUP), with sire of rams included or excluded from the training population

\begin{tabular}{lccccc}
\hline & \multicolumn{2}{c}{ Pseudo-ssGBLUP } & & \multicolumn{2}{c}{ Pseudo-BLUP } \\
\cline { 2 - 2 } \cline { 5 - 6 } & \multicolumn{2}{c}{ Sires of validation rams } & & \multicolumn{2}{c}{ Sires of validation rams } \\
\cline { 2 - 3 } \cline { 5 - 6 } Trait & Included & Excluded & & Included & Excluded \\
\hline Milk yield & 0.43 & 0.38 & & 0.32 & 0.28 \\
Fat percentage & 0.65 & 0.55 & & 0.58 & 0.44 \\
Protein percentage & 0.62 & 0.51 & & 0.54 & 0.45 \\
SCS & 0.59 & 0.47 & & 0.49 & 0.34 \\
Teat angle & 0.58 & 0.45 & & 0.47 & 0.28 \\
Udder cleft & 0.61 & 0.52 & & 0.48 & 0.37 \\
Udder depth & 0.57 & 0.44 & & 0.47 & 0.30 \\
\hline
\end{tabular}


and genomic relationships (ssGBLUP) is equivalent to imputing missing genotypes for females with phenotypic records (Christensen and Lund, 2010); using such a matrix enlarges the training population. However, slopes are still 0.14 points lower than 1.00 and should be improved, or they will result in giving an unfair advantage to juvenile over older selection candidates. By weighting the genomic and pedigree relationships matrices, Aguilar et al. (2010) and Christensen et al. (2012) were able to obtain improved slopes and more consistent reliabilities. This is still open to research (Christensen, 2012).

Habier et al. (2010) pointed out that accuracy of genomic selection greatly depends on the degree of relationship between validation and training populations. In effect, a slight loss of accuracy was observed when excluding sires of validation rams. Such a change resulted in 0.10 and 0.20 lower accuracy for pseudo-ssGBLUP and pseudo-BLUP, respectively. Greater sensitivity to the additive relationship compared with that reported by Habier et al. (2010) might be explained by the smaller size of the training set and low LD (0.11 instead of 0.20 at $<100 \mathrm{kbp}$ ), and also by the different design: full data sets and random sampling was used for Habier et al. (2010) versus reduced data sets and date-based cutoff in our work. Habier et al. (2010) also observed an increase in accuracy using BayesB but this increase was only visible for relationships lower than 0.125 (e.g., if candidates were great-grandsons of progeny-tested males), which seems an unrealistic case. It was also possibly explained by the existence of the major gene diacylglycerol O-acyltransferase 1 (DGAT1).

Accounting for preselection of genomic candidates is of additional interest when using ssGBLUP. Even with low selection intensity (25\%) at moderate heritability, Patry and Ducrocq (2011) found deteriorated accuracy of GEBV after only 1 generation. For future genomic breeding schemes, a selection intensity of $25 \%$ or more might be practiced. The ssGBLUP method takes selection into account and therefore provides unbiased GEBV.

\section{CONCLUSIONS}

This study confirms that a low level of LD is detected with the 50,000-marker $(50 \mathrm{~K})$ chip in the Lacaune breed compared with the Holstein breed. In agreement with this, we assessed a gain in accuracy between 0.10 and 0.20 across traits and models. This is considerably lower than in dairy cattle. The technical parameters about the accuracy of genomic prediction in Lacaune sheep are now known. Routine evaluation in the event of genomic selection is still to be determined, but the ssGBLUP offers the most general framework, possibly with improvements to consider heterogeneity of variances within herds. In our case, it results in better accuracy and lowest bias at the same computational cost compared with a multi-step procedure. The current focus is on designing a genomic breeding scheme. First, technical and biological parameters such as number of rams or seasonality of AI need to be considered. The high cost of genotyping relative to the individual value of the animal (even using low-density chips and imputation) imposes a significant cost/benefit challenge, and one which makes optimization of use of genomic prediction in dairy sheep likely to be quite different from that in dairy cattle.

\section{ACKNOWLEDGMENTS}

This work benefited from financial support of the Agence Nationale de la Recherche (ANR)-SheepSNPQTL (Paris, France), ApisGene (Paris, France), Fonds Unique Interministériel (FUI)-Roquefort'in projects, Midi-Pyrénees region (France), Le Fonds européen de développement régional (FEDER), Aveyron and Tarn department (France), and the city of Rodez (France). We thank the 5 breeder partners of the Roquefort'in project, the genotyping platform LABOGENA (http:// www.labogena.fr/; Jouy-en-Josas, France), the bioinformatics support of SIGENAE (http://www.sigenae. org/; Toulouse, France), the computing facilities of the Centre de Traitement de l'Information Genetique (CTIG; Jouy-en-Josas, France), and the bioinformatics platform GenoToul (http://bioinfo.genotoul.fr/; Toulouse, France). We thank the 2 reviewers for their constructive comments. Ignacy Misztal, Shogo Tsuruta (University of Georgia, Athens), and Ignacio Aguilar [Instituto Nacional de Investigación Agropecuaria (INIA), Canelones, Uruguay] answered many questions related to the use of software. Manuscript review by S. M. Hubbard of the Animal Improvement Programs Laboratory (US Department of Agriculture-Agricultural Research Service, Beltsville, MD) is gratefully acknowledged.

\section{REFERENCES}

Aguilar, I., I. Misztal, D. L. Johnson, A. Legarra, S. Tsuruta, and T. J. Lawlor. 2010. Hot topic: A unified approach to utilize phenotypic, full pedigree, and genomic information for genetic evaluation of Holstein final score. J. Dairy Sci. 93:743-752.

Aguilar, I., I. Misztal, A. Legarra, and S. Tsuruta. 2011. Efficient computation of the genomic relationship matrix and other matrices used in single-step evaluation. J. Anim. Breed. Genet. 128:422428.

Barillet, F. 1997. Genetics of milk production. Pages 539-564 in The Genetics of Sheep. I. Piper and A. Ruvinsky, ed. CAB International, Oxon, UK.

Barillet, F., J. M. Astruc, and G. Lagriffoul. 2007. Taking into account functional traits in dairy sheep breeding programs through the 
French example. Pages 57-64 in Breeding, Production Recording, Health and the Evaluation of Farm Animals. European Association for Animal Production (EAAP) Publ. No. 121. J. Kyntäjä, K. Lampinen, A. Rosati, and C. Mosconi, ed. Wageningen Academic Publishers, Wageningen, the Netherlands.

Barillet, F., J. M. Elsen, and F. Roussely. 1986. Optimization of a selection scheme for milk composition and yield in milk yield: Example of the Lacaune breed. Pages 658-664 in Proc. 3rd World Congr. Genet. Appl. Livest. Prod., Lincoln, NE. University of Nebraska, Lincoln.

Boichard, D. 2002. PEDIG: A Fortran package for pedigree analysis suited for large populations. Comm. 28-13 in Proc. 7th World Congr. Genetics Appl. Livest. Prod., Montpellier, France. INRA, Castanet-Tolosan, France.

Boichard, D., L. Maignel, and É. Verrier. 1997. The value of using probabilities of gene origin to measure genetic variability in a population. Genet. Sel. Evol. 29:5-23.

Browning, S. R., and B. L. Browning. 2007. Rapid and accurate haplotype phasing and missing-data inference for whole-genome association studies by use of localized haplotype clustering. Am. J. Hum. Genet. 81:1084-1097.

Buisson, D., J. M. Astruc, G. Baloche, X. Aguerre, P. Boulenc, F. Fidelle, B. Giral, P. Guibert, P. Panis, and F. Barillet. 2013. Assessment of technical and economic efficiency of genomic-based breeding programs in dairy sheep in France. Page 369 in Book of Abstracts of the 64th Annual Meeting of the European Federation of Animal Science, Nantes, France. Wageningen Academic Publishers, Wageningen, the Netherlands.

Christensen, O. F. 2012. Compatibility of pedigree-based and marker-based relationship matrices for single-step genetic evaluation. Genet. Sel. Evol. 44:37.

Christensen, O. F., and M. S. Lund. 2010. Genomic prediction when some animals are not genotyped. Genet. Sel. Evol. 42:2.

Christensen, O. F., P. Madsen, B. Nielsen, T. Ostersen, and G. Su. 2012. Single-step methods for genomic evaluation in pigs. Animal $6: 1565-1571$

Daetwyler, H. D., R. Pong-Wong, B. Villanueva, and J. A. Woolliams. 2010. The impact of genetic architecture on genome-wide evaluation methods. Genetics 185:1021-1031.

de Roos, A. P. W., B. J. Hayes, R. J. Spelman, and M. E. Goddard. 2008. Linkage disequilibrium and persistence of phase in HolsteinFriesian, Jersey and Angus cattle. Genetics 179:1503-1512.

Duchemin, S. I., C. Colombani, A. Legarra, G. Baloche, H. Larroque, J. M. Astruc, F. Barillet, C. Robert-Granié, and E. Manfredi. 2012. Genomic selection in the French Lacaune dairy sheep breed. J. Dairy Sci. 95:2723-2733.

Fikse, W. F., and G. Banos. 2001. Weighting factors of sire daughter information in international genetic evaluations. J. Dairy Sci. 84:1759-1767.

Gao, H., O. Christensen, P. Madsen, U. Nielsen, Y. Zhang, M. Lund, and G. Su. 2012. Comparison on genomic predictions using three GBLUP methods and two single-step blending methods in the Nordic Holstein population. Genet. Sel. Evol. 44:8.

Goddard, M. 2009. Genomic selection: Prediction of accuracy and maximisation of long term response. Genetica 136:245-257.

Gutiérrez, J. P., I. Cervantes, A. Molina, M. Valera, and F. Goyache. 2008. Individual increase in inbreeding allows estimating effective sizes from pedigree. Genet. Sel. Evol. 40:359-378.

Habier, D., J. Tetens, F.-R. Seefried, P. Lichtner, and G. Thaller. 2010. The impact of genetic relationship information on genomic breeding values in German Holstein cattle. Genet. Sel. Evol. 42:5.

Hayes, B. J., P. M. Visscher, H. C. McPartlan, and M. E. Goddard. 2003. Novel multilocus measure of linkage disequilibrium to estimate past effective population size. Genome Res. 13:635-643.

Hill, W. G. 1981. Estimation of effective population size from data on linkage disequilibrium. Genet. Res. 38:209-216.

Hill, W. G., and A. Robertson. 1968. Linkage disequilibrium in finite populations. Theor. Appl. Genet. 38:226-231.

International Sheep Genomics Consortium. 2010. International Sheep Genomics Consortium. Accessed Jan. 20, 2013. http://www.sheephapmap.org/.
Kijas, J. W., J. A. Lenstra, B. Hayes, S. Boitard, L. R. Porto Neto, M. San Cristobal. B. Servin, R. McCulloch, V. Whan, K. Gietzen, S. Paiva, W. Barendse, E. Ciani, H. Raadsma, J. McEwan, and B. Dalrymple., and other members of the International Sheep Genomics Consortium. 2012. Genome-wide analysis of the world's sheep breeds reveals high levels of historic mixture and strong recent selection. PLoS Biol. 10:e1001258.

Legarra, A., I. Aguilar, and I. Misztal. 2009. A relationship matrix including full pedigree and genomic information. J. Dairy Sci. 92:4656-4663.

Legarra, A., and V. Ducrocq. 2012. Computational strategies for national integration of phenotypic, genomic, and pedigree data in a single-step best linear unbiased prediction. J. Dairy Sci. 95:46294645 .

Mäntysaari, E., and M. Koivula. 2012. GEBV validation test revisited. Interbull Bull. 45:1-5.

Mäntysaari, E., Z. Liu, and P. VanRaden. 2010. Interbull validation test for genomic evaluations. Interbull Bull. 41:17-21.

Marie-Etancelin, C., J. M. Astruc, D. Porte, H. Larroque, and C. Robert-Granié. 2005. Multiple-trait genetic parameters and genetic evaluation of udder-type traits in Lacaune dairy ewes. Livest. Prod. Sci. 97:211-218.

Meadows, J. R. S., E. K. F. Chan, and J. W. Kijas. 2008. Linkage disequilibrium compared between five populations of domestic sheep. BMC Genet. 9:61.

Meuwissen, T. H. E., B. J. Hayes, and M. E. Goddard. 2001. Prediction of total genetic value using genome-wide dense marker maps. Genetics 157:1819-1829.

Misztal, I., S. Tsuruta, I. Aguilar, A. Legarra, P. M. VanRaden, and T. J. Lawlor. 2013. Methods to approximate reliabilities in single-step genomic evaluation. J. Dairy Sci. 96:647-654.

Misztal, I., S. Tsuruta, T. Stabel, B. Auvray, T. Druet, and D. H. Lee. 2002. BLUPF90 and related programs (BGF90). Commun. No. 28-07 in Proc. 7th World. Congr. Genet. Appl. Livest. Prod., Montpellier, France. INRA, Castanet-Tolosan, France.

Ober, U., J. F. Ayroles, E. A. Stone, S. Richards, D. Zhu, R. A. Gibbs, C. Stricker, D. Gianola, M. Schlather, T. F. C. Mackay, and H. Simianer. 2012. Using whole-genome sequence data to predict quantitative trait phenotypes in Drosophila melanogaster. PLoS Genet. 8:e1002685.

Olson, K. M., P. M. VanRaden, M. E. Tooker, and T. A. Cooper. 2011. Differences among methods to validate genomic evaluations for dairy cattle. J. Dairy Sci. 94:2613-2620.

Palhiere, I., F. Barillet, J. M. Astruc, X. Aguerre, J. P. Belloc, M. Briois, G. Fregeat, B. Bibe, H. Rochambeau, and D. Boichard. 2000. Analyse de la variabilité génétique des races ovines laitières Basco-Béarnaise, Lacaune et Manech à partir des informations généalogiques. Rencontres Recherches Ruminants 7:153-156.

Patry, C., and V. Ducrocq. 2011. Evidence of biases in genetic evaluations due to genomic preselection in dairy cattle. J. Dairy Sci. 94:1011-1020.

Pritchard, J. K., and M. Przeworski. 2001. Linkage disequilibrium in humans: Models and data. Am. J. Hum. Genet. 69:1-14.

Quittet, E., and M. Franck. 1983. Races ovines en France. Troisième edition. Mise à jour. La Maison Rustique, Paris, France.

Robert-Granié, C., B. Bonaïti, D. Boichard, and A. Barbat. 1999. Accounting for variance heterogeneity in French dairy cattle genetic evaluation. Livest. Prod. Sci. 60:343-357.

Rogers, A. R., and C. Huff. 2009. Linkage disequilibrium between loci with unknown phase. Genetics 182:839-844.

Sargolzaei, M., F. S. Schenkel, G. B. Jansen, and L. R. Schaeffer. 2008. Extent of linkage disequilibrium in Holstein cattle in North America. J. Dairy Sci. 91:2106-2117.

Schaeffer, L. R. 2006. Strategy for applying genome wide selection in dairy cattle. J. Anim. Breed. Genet. 123:218-223.

Sullivan, P. G. 2007. MTEDC software available for standardized EDC calculations. Interbull Bull. 37:78-81.

Sved, J. A. 1971. Linkage disequilibrium and homozygosity of chromosome segments in finite populations. Theor. Popul. Biol. 2:125141 
VanRaden, P. M. 2008. Efficient method to compute genomic predictions. J. Dairy Sci. 91:4414-4423.

VanRaden, P. M., C. P. Van Tassel, G. R. Wiggans, T. S. Sonstegard, R. D. Schnabel, J. F. Taylor, and F. S. Schenkel. 2009. Invited review: Reliability of genomic predictions for North American Holstein bulls. J. Dairy Sci. 92:16-24.
VanRaden, P. M., and G. R. Wiggans. 1991. Derivation, calculation, and use of national animal model information. J. Dairy Sci. 74:2737-2746.

Vitezica, Z. G., I. Aguilar, I. Misztal, and A. Legarra. 2011. Bias in genomic predictions for populations under selection. Genet. Res. (Camb.) 93:357-366. 